SCIREA Journal of Physics

ISSN: $2706-8862$

http://www.scirea.org/journal/Physics

April 19, 2021

SCIREA

Volume 6, Issue 1, February 2021

\title{
Rigid Motion in Special Relativity
}

\author{
Stuart Boehmer \\ Wichita, Kansas, United States \\ Email: seboehmer@shockers.wichita.edu; stuartboehmer@hotmail.com
}

\begin{abstract}
We solve the problem of rigid motion in special relativity in completeness, forswearing the use of the 4-D geometrical methods usually associated with relativity, for pedagogical reasons. We eventually reduce the problem to a system of coupled linear nonhomogeneous ordinary differential equations. We find that any rotation of the rigid reference frame must be independent of time. We clarify the issues associated with Bell's notorious rocket paradox and we discuss the problem of hyperbolic motion from multiple viewpoints. We conjecture that any rigid accelerated body must experience regions of shock in which there is a transition to fluid motion, and we discuss the hypothesis that the Schwarzchild surface of a black hole is just such a shock front.
\end{abstract}

Keywords : rigid body; rigid motion; born rigidity; rotation; acceleration; revolution; Ehrenfest's paradox; Bell's rocket paradox; relativity; hyperbolic motion; Schwarzchild solution; black holes 


\section{Introduction}

In 1910, Born [1] posed the problem of finding the most general rigid motion in special relativity. That is, he wanted to find the relativistic analogue of the classical,

$$
\boldsymbol{v}(\boldsymbol{x}, t)=\boldsymbol{V}(t)+\boldsymbol{\omega}(t) \times \boldsymbol{x},
$$

where $\boldsymbol{V}$ is the translational velocity and $\boldsymbol{\omega}$ is the rotational velocity of the moving rigid reference frame and $\boldsymbol{v}$ is the local velocity of the moving rigid frame with respect to the "absolute" frame of reference of Newton, and where $\boldsymbol{x}$ and $t$ are the Cartesian coordinates and time of the absolute frame. Of course, Born didn't intend to refer the relativistic solution to an absolute space, but to an arbitrary but fixed inertial reference frame, $\Sigma$.

Born solved the problem only incompletely and the complete solution seems to have remained an open problem to this day, 110 years later. In this paper, we find that the equivalent of (1) is an implicit algebraic relation where $\boldsymbol{v}$ appears on both sides of the equation.

In order to solve the trajectories of points fixed in the moving rigid frame, one must solve the system of coupled linear nonhomogeneous ordinary differential equations,

$$
\frac{d x}{d t}-\boldsymbol{\omega}(t) \times \boldsymbol{x}=\boldsymbol{V}(t)
$$

where $\boldsymbol{\omega}$ and $\boldsymbol{V}$ are given functions of $t$, determined by a central trajectory of interest (this will be discussed in detail in $\S 5$ where we discuss the " $C_{0}$ problem"). The overall situation remains the same in the relativistic case, as we shall see.

Just what do we mean when we say a reference frame, whether inertial (here denoted by $\Sigma$ ) or accelerated and rotated (here denoted by $\Upsilon$ ), is "rigid"? Just this, that the six mutual distances between any non-coplanar tetrad of points fixed in the reference frame within a differential neighborhood of one another are constant in time. If not, the generic reference frame (denoted also by the symbol, $\Upsilon$ ) is said to be in a general fluid motion. We do not consider such fluid reference frames further in this paper except indirectly.

If the spatial coordinates in $\Upsilon$ are $\boldsymbol{u}^{\prime}$ and the time variable is $t^{\prime}$, then $\boldsymbol{u}^{\prime}$ are constants for points fixed in $\Upsilon$, whether $\Upsilon$ is rigid or not (and we allow for the possibility of curvilinear, non-Cartesian coordinates, while time is the proper time or clock readings for points fixed in $\Upsilon$, regarded as trajectories in $\Sigma$ if necessary). If we consider four such points, $\boldsymbol{u}_{K}^{\prime}, K=$ $(1,2,3,4)$, which are in the same differential neighborhood and non-coplanar, then there are 
eighteen constant differential quantities, $d u_{m, K 1, K 2}^{\prime}:=u_{m, K 1}^{\prime}-u_{m, K 2}^{\prime}$, defining six mutual distances, $d s_{K 1, K 2}^{2}=g_{m n}^{\prime} d u_{m, K 1, K 2}^{\prime} d u_{n, K 1, K 2}^{\prime}$, where $m$ and $n$ are summed 1 to $3, g_{m n}^{\prime}$ is the spatial metric of $\Upsilon$ and $d s$ is the spatial distance (in this paper we denote the four-metric by $\mathrm{g}$ and the four-element by $d \sigma)$. Since the eighteen quantities, $d u_{m, K 1, K 2}^{\prime}$, are constant, a necessary and sufficient condition of the six $d s_{K 1, K 2}$ being constant is that the six independent $g_{m n}^{\prime}$ be constant in time, or that $\frac{\partial g_{m n}^{\prime}}{\partial t^{\prime}}=0$ - the Born rigidity condition.

Let us digress for a moment. Born was fully aware that there can be no rigid bodies in relativity because the effects of a locally applied torque or force could not propagate faster than speed $c$ and plastic flow would necessarily ensue. However a rigid motion or reference frame might still be conceptually possible because such a motion might arise fortuitously over small regions of space for brief periods of time, or perhaps as the result of the use of relays in some fashion to orchestrate a pre-programmed series of motions. For that matter, rigid bodies are not possible in classical theory because the Young's modulus of no real material can be infinite (though of course a complete theory of the properties of real materials must rely on quantum theory). Nevertheless, the abstract study of rigid motion or reference frames remains worthy of our consideration. Possible or not, rigid bodies do not occur in nature, but that doesn't forbid the postulation of the existence of rigid frames of reference such as the inertial reference frames, $\Sigma$, of special relativity are assumed to be.

Since in a rigid frame, we've, $\partial g_{m n}^{\prime} / \partial t^{\prime}=0$, clearly the spatial curvature of a rigid frame cannot change with time (though of course it may vary spatially from one point, $\boldsymbol{u}^{\prime}$, to another). This means that if a reference frame is initially spatially Euclidean (zero spatial curvature), it must remain so and similarly if it has a certain nonzero curvature at $\boldsymbol{u}^{\prime}$. Note however that the four-curvature of any reference frame (even fluid ones) in special relativity must of course be identically zero at all places and times, but here we are talking about the spatial or three-curvature, which is related to the Riemann-Christoffel 3-tensor calculated from the 3-metric, $g_{m n}^{\prime}$, in the usual way. Such a quantity (like $g_{m n}^{\prime}$ for that matter) is of course not a four-tensor, but we do not restrict ourselves to the consideration of such quantities in this paper. The theory is still relativistically covariant, though transformation laws for quantities may be of a novel kind. The velocity addition law for three-velocities is also not of the usual tensorial nature, which does not prevent it from being relativistically covariant. 
Now, a modification of a succinct argument made by Ehrenfest [2] indicates that a rotating reference frame must be non-Euclidean. It runs like this: The circumference and radius of a rotating disk at rest in the rotating frame, $\Upsilon$, are $C^{\prime}, R^{\prime}$. The inertial frame, $\Sigma$, which is at rest with respect to the center of rotation is in motion with respect to the circumference, $C$, of radius, $R$, and therefore must experience a Lorentz contraction, $C<C^{\prime}$, but the radius is normal to the direction of motion and experiences no contraction, $R=R^{\prime}$. Now the postulate of special relativity is that $\Sigma$ is always Euclidean, $\frac{C}{R}=2 \pi$; therefore, $\frac{C^{\prime}}{R^{\prime}}>2 \pi$, and $\Upsilon$ has negative spatial curvature which depends on the rotation rate, $\omega$, and $R^{\prime}$ or $R$, because the magnitude of the Lorentz contraction, $C / C^{\prime}$, depends on the velocity, $\omega R$, of the circumference. (Incidentally, Ehrenfest originally presented his results as a paradox since he assumed that $\Upsilon$ as well as $\Sigma$ must be Euclidean and this was contradictory. Apparently Ehrenfest at the time was simply unfamiliar with the existence of non-Euclidean geometries.) Thus, because of the necessary temporal constancy of the spatial curvature, the rotation rate of a rotating disk or reference frame must also be a temporal constant, though it may depend on spatial position. In $\S 5$, we will find, through formal mathematical manipulation of certain differential operators that recur throughout the theory, that a condition of rigidity (implicit in the Born rigidity condition) is in fact, $\partial \omega / \partial t=0$.

We will find that a rigid reference frame can accelerate without rotation in a completely arbitrary fashion. Thus it is Euclidean because we may start out with it at rest with respect to $\Sigma$, which is Euclidean, and accelerate to any desired permissible (rigid) state. Since it is Euclidean, we may find Cartesian coordinates for it; in fact the simplest choice is just $\boldsymbol{u}^{\prime}=$ $\boldsymbol{x}_{0}$, where $\boldsymbol{x}_{0}$ are the Cartesian coordinates of $\Sigma$ coinciding with the point in $\Upsilon$ with which it is temporarily at rest. Because $g_{m n}^{\prime}$ initially equals $\delta_{m n}$ for these $\boldsymbol{u}^{\prime}$, it will remain so for all time $\left(\partial g_{m n}^{\prime} / \partial t^{\prime}=0\right)$ and $\boldsymbol{u}^{\prime}$ will therefore remain Cartesian for all time. Thus, a rigid frame in hyperbolic motion can be described by such Cartesian coordinates. This case is discussed by Misner, Thorne and Wheeler [3] and we will beat it to death for illustrative purposes in this paper because it is readily solvable and "the artist paints what he is able", to quote Nietzsche.

Next, let us consider the case of Bona [4], a researcher who invented an alternative theory of relativity in which arbitrary rotation was permissible. Apparently he was motivated by a desire to facilitate theoreticians whom wanted to develop a non-point particle theory of the electron in which an arbitrarily rotating electron was nonetheless rigid. It is clear from 
Ehrenfest's argument given above that in such a theory the rotating disk must be Euclidean, $\frac{C^{\prime}}{R^{\prime}}=2 \pi$; that is, there can be no Lorentz contraction along the circumference. Perhaps Bona was thinking that the Lorentz contraction was rotation-dependent, being present for translational motion, but absent for rotational motion, even of the same total $v$. However, this is untenable as can be seen form considering the following example. Suppose we consider the limiting case, $\omega \rightarrow 0, R \rightarrow \infty, v=\omega R=$ const., which is indistinguishable from translational motion where a Lorentz contraction must be present; Bona's theory is consistent only if there is no Lorentz contraction for translation either. Nevertheless Bona attempts to give his theory substance by giving a physically awkward and apparently inconsistent interpretation to the 4line-element and the 4-metric, $\mathfrak{g}_{\mu \nu}$ (in this paper, Greek indices range 1-4; Latin, 1-3), in a rotating reference frame following Selleri [5], Klauber [5] and others with similar motivations. (It is probably inconsistent, because it leads to a Lorentz contraction for translational motion but not the equivalent rotational motion.)

Bona solves his problem as posed fully analytically and cites Synge [6] as the authority to consult for the usual theory. However, consulting this reference I find no indication of a complete solution, just a general discussion of symmetry relations.

As a final topic in this introduction, let us discuss the methodology of this paper.

As already intimated, we do not employ the 4-D geometrical methods usually associated with relativity (seeking out 4-vectors and tensors, etc.) because it is our wish to proceed by giving direct physical meaning to the coordinates in any reference frame, $\Sigma$ or $\Upsilon$, rather than adopting the fashionable viewpoint - going back to Einstein [7] - that it is not important to give physical meaning to coordinates, which are just convenient overlays on the physical problem at hand. It is our belief that giving the coordinates direct physical meaning ( $t^{\prime}$ is a clock reading at a specific point, $\boldsymbol{u}^{\prime}$, in $\Upsilon ; \boldsymbol{u}^{\prime}$ are coordinates referring exclusively to spacei.e., serving to distinguish different observers at rest in $\Upsilon$ ) has great pedagogical value. Moreover, the question of rigidity, dealing as it does with spatial distance, is naturally understood in such 3-D terms where "time" is something that flows through "space", rather than space and time being part of some inseparable whole - it is a less hyperopic and more intelligible approach. This is not to say that we cannot use transformations of the coordinates to solve any given physical problem in a mathematically succinct way-but we should regard these as mere mathematical artifices rather than associate with them any occult physical 
significance, as many authors are wont to do (especially in regards to a nonphysical choice for $u_{4}$ : if it is not the reading on some observer's clock then it is not any sort of "time").

There are other ways to discuss relativistic covariance than by the exclusive use of 4-vectors and tensors. That may be mathematically convenient and powerful, but it obscures the physical meaning of the theory.

Rather than hyperopically postulating the existence of an occult "space-time manifold" (which was the brainchild of Minkowski, not Einstein), let us learn to think of the space of a given reference frame as something through which time "flows". The interrelation between space and time is reference frame dependent-and there is some arbitrariness in the definition of a "reference frame" (it is not necessarily rigid, among other things). This interrelation between space and time is more "fluid" and physically interactive in relativistic theories than in the "absolute" conceptions of Newton (with which Newton himself was privately dissatisfied, though he would never publicly admit this to Leibnitz). The "boundaries" between space and time shift dynamically as a physical situation unfolds. Of course, these statements are mere artistic hand-waving, but we are trying to paint a picture, alternate to Minkowski's "block space-time", in which nature is instead something dynamic and evolving, as we all know it is. The space-time manifold concept is also deficit in the sense that it creates the anticipation that it is meaningful to discuss time on a global scaleuniversal time, reminiscent of Newton's absolute time. It is not: the experience of time is exclusively a local affair, a fact which we turn now to discuss.

In relativistic theories, meaningful discussions of the flow of time must be confined to individual clocks, observers or (what is the same thing) locations in space, since the essence of Einstein's 1905 insight was that any attempt to synchronize clocks across space must be based upon some arbitrary convention and it represents nothing physically real: different observers exist independently, each in his own private world of time. Transporting a clock slowly with respect to the reference frame of interest (so that Lorentzian time dilatation is minimized) to make a direct comparison of clocks at two different locations seems to be a real, objective process, but might fail for any number of reasons. For instance, if gravitational time dilatation is present, clocks initially synchronized would not remain so for long. Also, the Sagnac effect [5] presents similarly insurmountable difficulties in rotating and non-rigid reference frames - but an in-depth discussion of all this here would take us too far afield. 
This conception is opposed to the pedagogical approach to the "space-time manifold" which has unfortunately become standard, which runs something as follows:-

Time is a "direction" in space-time and space is "perpendicular" to it. At each point in spacetime there is a light cone which defines the directions of future and past, while points lying outside the light cone are spacelike.

All this is nonsense and should not be taught as the basic concepts of space and time, but merely as derivative mathematical tools useful for proving abstract theorems concerning spatial and temporal relations.

The proper conception is powerful in its simplicity and facilitates a proper understanding of the abstract theorems and powerful mathematical tools associated with relativity. A point of "space" is simply a given observer, specified by the spatial coordinates, $\boldsymbol{u}$ ', of some reference frame, while "time" is simply the local clock reading of this observer. To repeat: time is an exclusively local affair, a private experience of the individual observer, measured by a clock, and it is not a "direction" in some vague "space-time manifold". Anyone not in command of these basic facts will be led afield by machinery which is too powerful for him to control skillfully.

Lastly, the fact that here we do not avail ourselves of the usual 4-D geometrical techniques probably makes our approach novel, unique and worthy of publication even if the problem has been solved elsewhere. If nothing else, it serves to illustrate the continuity between classical and relativistic conceptions of reality, which was a large part of our purpose.

\section{Derivation of the Equations of Rigid Motion.}

Given an inertial reference frame, $\Sigma$, with spatial coordinates, $\boldsymbol{x}$, and time, $t$, and the velocity, $\boldsymbol{v}(\boldsymbol{x}, t)$, at each point of $\Sigma$ of some other arbitrary reference frame, $\Upsilon$, (perhaps fluid), we must calculate the spatial metric, $g_{m n}^{\prime}$, of $\Upsilon$ in its own coordinate system, $\boldsymbol{u}^{\prime}$, as a function of the time, $t^{\prime}$, of $\Upsilon$. If $\Upsilon$ is fluid, $\boldsymbol{u}^{\prime}$ must be curvilinear, and there is no reason they cannot be even if $\Upsilon$ is rigid, so we allow for this general possibility.

From the transformations, $(\boldsymbol{x}, t)=\left(\boldsymbol{x}\left(\boldsymbol{u}^{\prime}, t^{\prime}\right), t\left(\boldsymbol{u}^{\prime}, t^{\prime}\right)\right)$, we can calculate the spatial distance in $\Upsilon$ from the coordinates of $\Sigma$ and the velocity, $v$, at each point in $\Sigma$. Let us first illustrate the procedure for classical theory; the steps in the relativistic theory are exactly the same, 
complicated only by the fact that the Lorentz transformation is more complicated than the Galilean.

Suppose first that $\Upsilon$ is another inertial frame specified by a $v$ which is spatially and temporally constant. Then we can use Cartesian coordinates in $\Upsilon$, and,

$$
\begin{aligned}
& x_{i}^{\prime}=x_{i}-v_{i} t, \\
& t^{\prime}=t .
\end{aligned}
$$

Now, the distance in $\Upsilon$ is given by, $d s^{\prime 2}=d x_{m}^{\prime} d x_{m}^{\prime}$, (Einstein summation convention on repeated indices). Therefore, in terms of the coordinates and time of $\Sigma$,

$$
d s^{\prime 2}=d x_{m} d x_{m}-2 v_{m} d x_{m} d t+v^{2} d t^{2}
$$

where $v^{2}:=v_{m} v_{m}$

We can write this as,

$$
d s^{2}=\varphi_{\mu \nu} d x_{\mu} d x_{v}
$$

where,

$$
\varphi_{m n}=\delta_{m n}, \quad \varphi_{m 4}=\varphi_{4 m}=-v_{m}, \quad \varphi_{44}=v^{2},
$$

and $d x_{4}:=d t$

In (1) we have the expression for $d s^{\prime 2}$ in terms of the coordinates of $\Sigma$ and the velocity.

Note that,

$$
\begin{gathered}
\varphi_{m 4}=\varphi_{4 m}=-v_{n} \varphi_{m n} \\
\varphi_{44}=-v_{m} \varphi_{4 m}=v_{m} v_{n} \varphi_{m n}
\end{gathered}
$$

The result (3), (3') is valid in relativity too; its significance will be explained presently.

As derived, (1) and (2) are valid only if $\Upsilon$ is an inertial (and therefore also rigid) reference frame with Cartesian coordinates, $\boldsymbol{x}$. A key step at this juncture is to invoke the principle of locality; that is, we now assume that (1), (2) are valid even if $\Upsilon$ is a general (possibly fluid or non-rigid) reference frame. That is, we assume $\varphi_{\mu \nu}$ is the same function of the local velocity even if $\Upsilon$ is globally non-rigid. This is justifiable because part of the definition of the concept of a "reference frame" is that all points in a differential neighborhood are separating from one another at no more than differential rates - i.e., they all have the same velocity with respect to $\Sigma$ to within a differential amount - so that any ambiguity in the definition of distances 
between them is no more than of second order (viz.: proportional to $d v d u$ ). That is, if two points in the same differential neighborhood have a finite velocity with respect to one another, they cannot be regarded as being part of the same reference frame. Thus, for instance, the thermodynamic motions of neighboring molecules in the atmosphere cannot be consolidated into a single consistent reference frame. This obviates the difficulty that would arise in relativistic theories with Lorentz contractions that the "distance" between two particles, even as judged in the reference frame of the particles themselves may depend on which particle is making the measurement; the difficulty arises even if the two particles are in differential relative motion of course, but leads to only an ambiguity of second order.

Now, for general $\Upsilon$, we need an expression for $g_{m n}^{\prime}$ whose components refer to the coordinates of $\Upsilon$ derived from the quantities in (1) and (2).

We assume that in $\Upsilon$, even if it is non-rigid, $d s^{\prime}$ is a purely spatial quantity; that is, it depends on only the differentials of the spatial coordinates, $d \boldsymbol{u}^{\prime}$. Thus, we assume $g^{\prime}$ has components $g_{m n}^{\prime}$ only for $(m, n)$ ranging $1-3$, though note that these components are in general functions of $t^{\prime}$ as well as $\boldsymbol{u}^{\prime}$ because it is only in rigid frames that $\partial g_{m n}^{\prime} / \partial t^{\prime}=0$.

Thus, we assume that,

$$
d s^{\prime 2}=g_{m n}^{\prime} d u_{m}^{\prime} d u_{n}^{\prime}
$$

In particular, the distance between a fixed point, $\boldsymbol{u}^{\prime}$, and itself is zero; i.e. $d s^{\prime}=0$ when $d \boldsymbol{u}^{\prime}=0$.

Now,

$$
\begin{gathered}
d x_{m}=\frac{\partial x_{m}}{\partial u_{r}^{\prime}} d u_{r}^{\prime}+\frac{\partial x_{m}}{\partial t^{\prime}} d t^{\prime}, \\
d t=d t^{\prime} .
\end{gathered}
$$

Note that $\frac{\partial x_{m}}{\partial t^{\prime}}$ means $\left.\frac{\partial x_{m}}{\partial t^{\prime}}\right|_{\boldsymbol{u}^{\prime}}$, which, since $d t^{\prime}=d t$, is the velocity of a point, $\boldsymbol{u}^{\prime}$, fixed in $\Upsilon$ with respect to $\Sigma$; i.e.: $v_{m}$. Thus,

$$
d x_{m}=\frac{\partial x_{m}}{\partial u_{r}^{\prime}} d u_{r}^{\prime}+v_{m} d t^{\prime}
$$

That is, when $d u_{r}^{\prime}=0, d x_{m}=v_{m} d t^{\prime}$; using this in (1) and setting $d s^{\prime}=0$, we've,

$$
\varphi_{m n} v_{m} v_{n}+\varphi_{m 4} v_{m}+\varphi_{4 m} v_{m}+\varphi_{44}=0
$$

which is satisfied identically using (3). 
Thus, we have, as promised, discovered the significance of (3): it is due to the fact that the spatial distance, $d s^{\prime}$, in $\Upsilon$ depends on only the spatial differentials, $d \boldsymbol{u}^{\prime}$, and not also on $d t^{\prime}$.

Now, substituting (5) (without $d \boldsymbol{u}^{\prime}=0$ ) in (1), recalling (2) and (3), we obtain,

$$
d s^{\prime 2}=\frac{\partial x_{m}}{\partial u_{r}^{\prime}} \frac{\partial x_{m}}{\partial u_{s}^{\prime}} d u_{r}^{\prime} d u_{s}^{\prime}
$$

or, recalling (4),

$$
g_{r s}^{\prime}=\frac{\partial x_{m}}{\partial u_{r}^{\prime}} \frac{\partial x_{m}}{\partial u_{s}^{\prime}}
$$

which is reminiscent of the usual relation for coordinate transformations in ordinary 3geometry; but recall that there is a different meaning here because the transition from $\boldsymbol{x}$ to $\boldsymbol{u}$ involves a change of reference frame, i.e. a velocity boost, something that of course does not occur in a time-less 3-geometry.

Differentiating (6) with respect to $t^{\prime}$, recalling the rigidity condition, and that $\frac{\partial x_{m}}{\partial t^{\prime}}=v_{m}$, we've,

$$
0=\frac{\partial v_{m}}{\partial u_{r}^{\prime}} \frac{\partial x_{m}}{\partial u_{s}^{\prime}}+\frac{\partial x_{m}}{\partial u_{r}^{\prime}} \frac{\partial v_{m}}{\partial u_{s}^{\prime}}
$$

Now, $\frac{\partial}{\partial u_{r}^{\prime}}=\frac{\partial x_{k}}{\partial u_{r}^{\prime}} \frac{\partial}{\partial x_{k}}+\frac{\partial t}{\partial u_{r}^{\prime}} \frac{\partial}{\partial t}=\frac{\partial x_{k}}{\partial u_{r}^{\prime}} \frac{\partial}{\partial x_{k}}$ (because $t=t^{\prime}$, therefore $\left.\frac{\partial t}{\partial u_{r}^{\prime}}=0\right)$, while $\delta_{k l}=\frac{\partial x_{k}}{\partial u_{r}^{\prime}} \frac{\partial u_{r}^{\prime}}{\partial x_{l}}+$ $\frac{\partial x_{k}}{\partial t^{\prime}} \frac{\partial t^{\prime}}{\partial x_{l}}=\frac{\partial x_{k}}{\partial u_{r}^{\prime}} \frac{\partial u_{r}^{\prime}}{\partial x_{l}}$, therefore, multiplying by $\frac{\partial u_{r}^{\prime}}{\partial x_{k}} \frac{\partial u_{s}^{\prime}}{\partial x_{l}}$ and summing on $r$ and $s$, we obtain,

$$
\frac{\partial v_{k}}{\partial x_{l}}+\frac{\partial v_{l}}{\partial x_{k}}=0
$$

Thus, we've expressed the rigidity condition for $\Upsilon$ in terms of the coordinates of $\Sigma$ and the relative velocity as measured by $\Sigma$ at that point. This is the result we have been seeking, with which we will conclude the discussion of the classical rigidity condition for now; let us move on to the relativistic case for a similar result.

In the relativistic case, in order to derive the expression for $\varphi_{\mu \nu}$ one must use the Lorentz transformation instead of the Galilean in the first step. One can use the Lorentz transformation for a general direction of $\boldsymbol{v}$, which can be found in Tsamparlis [8], or one can use the usually expressed Lorentz transformation with $v$ along the $x$-axis followed by a spatial rotation. The final result in either case is,

$$
\varphi_{m n}=\delta_{m n}+\frac{v_{m} v_{n} \gamma^{2}}{c^{2}}
$$




$$
\begin{aligned}
\varphi_{m 4} & =\varphi_{4 m}=-v_{m} \gamma^{2}, \\
\varphi_{44} & =v^{2} \gamma^{2}
\end{aligned}
$$

where,

$$
\gamma^{2}:=\frac{1}{1-v^{2} / c^{2}}
$$

We see that (3) holds in this case too.

In this case, a different technique than in the classical case is convenient. Recall (1). Now suppose we use, rather than the $x_{\mu}$, a new set of arbitrary coordinates, $\widetilde{u}_{\mu}$, where, $x_{\mu}=x_{\mu}(u)$. Then,

$$
d s^{\prime 2}=\widetilde{\varphi}_{\mu \nu} d \widetilde{u}_{\mu} d \widetilde{u}_{v}
$$

where, $\widetilde{\varphi}_{\mu \nu}=\varphi_{\rho \sigma} \frac{\partial x_{\rho}}{\partial \widetilde{u}_{\mu}} \frac{\partial x_{\sigma}}{\partial \widetilde{u}_{v}}$, and, $\varphi_{\mu \nu}=\widetilde{\varphi}_{\rho \sigma} \frac{\partial \widetilde{u}_{\rho}}{\partial x_{\mu}} \frac{\partial \widetilde{u}_{\sigma}}{\partial x_{v}}$.

Suppose we choose $\widetilde{u}=\left(u_{m}^{\prime}, t^{\prime}\right)$, such that $\widetilde{\varphi}_{m n}=g_{m n}^{\prime}, \widetilde{\varphi}_{m 4}=\widetilde{\varphi}_{4 m}=\widetilde{\varphi}_{44}=0$ (because the velocity of $\Upsilon$ - the reference frame whose $d s^{\prime}$ is being calculated-with respect to the coordinates, $\widetilde{u}$, is zero); then,

$$
\varphi_{\mu \nu}=g_{m n}^{\prime} \frac{\partial u_{m}^{\prime}}{\partial x_{\mu}} \frac{\partial u_{n}^{\prime}}{\partial x_{v}}
$$

where now, $\varphi$ refers to the $d s^{\prime}$ of $\Upsilon$ in the coordinates, $x_{\mu}$, of $\Sigma$. Because both $g^{\prime}$ and $\varphi$ are of rank three (all components of $\varphi_{\mu \nu}$ are determined by $\varphi_{m n}$ through (3)), we need consider only $(\mu, v)=(r, s)$; hence, recalling $(9)$ :-

$$
\delta_{r s}+v_{r} v_{s} \gamma^{2} / c^{2}=g_{m n}^{\prime} \frac{\partial u_{m}^{\prime}}{\partial x_{r}} \frac{\partial u_{n}^{\prime}}{\partial x_{s}} .
$$

The rigidity condition is $\partial g_{m n}^{\prime} / \partial t^{\prime}=0$, so operating with $\partial / \partial t^{\prime}$, we've,

$$
\frac{\partial}{\partial t^{\prime}}\left(v_{r} v_{s} \gamma^{2} / c^{2}\right)=g_{m n}^{\prime}\left[\frac{\partial u_{n}^{\prime}}{\partial x_{s}} \frac{\partial}{\partial t^{\prime}}\left(\frac{\partial u_{m}^{\prime}}{\partial x_{r}}\right)+\frac{\partial u_{m}^{\prime}}{\partial x_{r}} \frac{\partial}{\partial t^{\prime}}\left(\frac{\partial u_{n}^{\prime}}{\partial x_{s}}\right)\right] \text {. }
$$

Now, $\frac{\partial}{\partial t^{\prime}}=\frac{\partial t}{\partial t^{\prime}} \frac{\partial}{\partial t}+\frac{\partial x_{m}}{\partial t^{\prime}} \frac{\partial}{\partial x_{m}}$. Moreover, $\frac{\partial x_{m}}{\partial t^{\prime}}$ means $\left.\frac{\partial x_{m}}{\partial t^{\prime}}\right|_{\boldsymbol{u}^{\prime}}=\left.\frac{\partial x_{m}}{\partial t}\right|_{\boldsymbol{u}^{\prime}} \frac{\partial t}{\partial t^{\prime}}$, where $\left.\frac{\partial x_{m}}{\partial t}\right|_{\boldsymbol{u}^{\prime}}$ is just the velocity of a point, $\boldsymbol{u}^{\prime}$, fixed in $\Upsilon$, i.e., $v_{m}$, and we've,

$$
\frac{\partial}{\partial t^{\prime}}=\frac{\partial t}{\partial t^{\prime}}\left(\frac{\partial}{\partial t}+v_{m} \frac{\partial}{\partial x_{m}}\right)
$$

Note that in (12) we therefore have a common factor of $\frac{\partial t}{\partial t}$ in all terms, which may be divided out. Note, too, that, 


$$
\left(\frac{\partial}{\partial t}+v_{k} \frac{\partial}{\partial x_{k}}\right)\left(\frac{\partial u_{m}^{\prime}}{\partial x_{r}}\right)=\frac{\partial^{2} u_{m}^{\prime}}{\partial x_{r} \partial t}+v_{k} \frac{\partial^{2} u_{m}^{\prime}}{\partial x_{k} \partial x_{r}}
$$

Now, $\frac{\partial u_{m}^{\prime}}{\partial t}$ may be determined from, $\frac{\partial u_{m}^{\prime}}{\partial t} \frac{\partial t}{\partial t^{\prime}}+\frac{\partial u_{m}^{\prime}}{\partial x_{k}} \frac{\partial x_{k}}{\partial t^{\prime}}=0$, or,$\frac{\partial t}{\partial t^{\prime}}\left(\frac{\partial u_{m}^{\prime}}{\partial t}+\frac{\partial u_{m}^{\prime}}{\partial x_{k}} v_{k}\right)=0$, or, $\frac{\partial u_{m}^{\prime}}{\partial t}=$ $-\frac{\partial u_{m}^{\prime}}{\partial x_{k}} v_{k}$

Substituting in the above, we therefore have,

$$
\left(\frac{\partial}{\partial t}+v_{k} \frac{\partial}{\partial x_{k}}\right)\left(\frac{\partial u_{m}^{\prime}}{\partial x_{r}}\right)=-\frac{\partial v_{k}}{\partial x_{r}} \frac{\partial u_{m}^{\prime}}{\partial x_{k}}
$$

and (12) becomes,

$$
0=\left(\frac{\partial}{\partial t}+v_{k} \frac{\partial}{\partial x_{k}}\right)\left(\frac{v_{r} v_{s} \gamma^{2}}{c^{2}}\right)+g_{m n}^{\prime}\left(\frac{\partial u_{n}^{\prime}}{\partial x_{s}} \frac{\partial u_{m}^{\prime}}{\partial x_{k}} \frac{\partial v_{k}}{\partial x_{r}}+\frac{\partial u_{m}^{\prime}}{\partial x_{r}} \frac{\partial u_{n}^{\prime}}{\partial x_{k}} \frac{\partial v_{k}}{\partial x_{s}}\right)
$$

Recalling (11), this becomes,

$$
0=\left(\frac{\partial}{\partial t}+v_{k} \frac{\partial}{\partial x_{k}}\right)\left(\frac{v_{r} v_{s} \gamma^{2}}{c^{2}}\right)+\varphi_{s k} \frac{\partial v_{k}}{\partial x_{r}}+\varphi_{r k} \frac{\partial v_{k}}{\partial x_{s}}
$$

Some algebraic manipulation yields,

$$
\left(L_{l} v_{k}+L_{k} v_{l}\right) \varphi_{r k} \varphi_{l s}=0
$$

where $L$ is the differential operator,

$$
L_{i}:=\frac{\partial}{\partial x_{i}}+\frac{v_{i}}{c^{2}} \frac{\partial}{\partial t}
$$

Because $\varphi_{m n}$ is rank three and has a nonzero determinant, (13) is equivalent to,

$$
L_{m} v_{n}+L_{n} v_{m}=0
$$

This is to be compared with the classical rigidity condition, (7). Note that (14) becomes (7) in the limit $c \rightarrow \infty$.

This concludes our discussion of the relativistic rigidity condition for now. Next we demonstrate that it is relativistically covariant.

\section{The Covariance of the Relativistic Rigidity Condition.}

So far, we have specialized the theory to an arbitrary but fixed inertial frame, $\Sigma$, to discuss the motion of $\Upsilon$. We must now show that transforming to a new inertial frame, $\Sigma$ ', gives the same 
equation, (14), when expressed in the coordinates, $\left(\boldsymbol{x}^{\prime}, t^{\prime}\right)$, and velocity, $\boldsymbol{v}^{\prime}$, as measured in $\Sigma^{\prime}$. Of course, the velocity, $\boldsymbol{V}$, of $\Sigma^{\prime}$ with respect to $\Sigma$ is constant, since both are inertial frames.

As a first step, we demonstrate that (14) is invariant under rotations of the spatial coordinates, $\boldsymbol{x}$; then we are free to choose $\boldsymbol{V}$ along the $x$-axis without loss of generality and thereby use the form traditionally given for the Lorentz transformation. (again, if desired, we could use the

Lorentz transformation for a general direction of $\boldsymbol{V}$, which is given by Tsamparlis [8].)

We give, without commentary, this demonstration, where $R$ is a rotation matrix and $\bar{R}$ is its inverse or transpose:-

$$
x_{n}=R_{. s}^{n} x_{s}^{\prime} ; \quad \frac{\partial}{\partial x_{n}}=\bar{R}_{. n}^{s} \frac{\partial}{\partial x_{s}^{\prime}} ; \quad v_{n}=R_{. s}^{n} v_{s}^{\prime} ; L_{n}=\bar{R}_{. n}^{s} \frac{\partial}{\partial x_{s}^{\prime}}+R_{. s}^{n} \frac{v_{s}^{\prime}}{c^{2}} \frac{\partial}{\partial t} ;
$$

but, $\bar{R}_{. n}^{s}=R_{. s}^{n}$, so, $L_{n}=R_{. s}^{n} L_{s}^{\prime}$, and,

$$
L_{m} v_{n}+L_{n} v_{m}=R_{. r}^{m} R_{. s}^{n}\left(L_{r}^{\prime} v_{s}^{\prime}+L_{s}^{\prime} v_{r}^{\prime}\right)
$$

therefore if the left hand side is zero, so is the expression in brackets, and vice versa, demonstrating invariance under spatial rotation.

Next, let us demonstrate covariance under a constant velocity boost of $\Sigma$ along the $x$-axis.

First consider the $m=n=1$ component of (14), viz., $L_{1} v_{1}=0$, or,

$$
\frac{\partial v_{x}}{\partial x}+\frac{v_{x}}{c^{2}} \frac{\partial v_{x}}{\partial t}=0
$$

Now, from the Lorentz transformation connecting $(x, t)$ and $\left(x^{\prime}, t^{\prime}\right)$, we can deduce,

$$
\begin{gathered}
\frac{\partial}{\partial x}=\gamma_{V}\left(\frac{\partial}{\partial x^{\prime}}-\frac{V}{c^{2}} \frac{\partial}{\partial t^{\prime}}\right), \\
\frac{\partial}{\partial t}=\gamma_{V}\left(\frac{\partial}{\partial t^{\prime}}-V \frac{\partial}{\partial x^{\prime}}\right),
\end{gathered}
$$

where, $\gamma_{V}:=\frac{1}{\sqrt{1-V^{2} / c^{2}}}$.

Now, the velocity addition law says,

$$
v_{x}=\frac{v_{x}^{\prime}+V}{1+v_{x}^{\prime} V / c^{2}} .
$$

Differentiating, because $V$ is constant, we've, 


$$
d v_{x}=\frac{d v^{\prime}{ }_{x}}{\gamma_{V}^{2}\left(1+v^{\prime}{ }_{x} V / c^{2}\right)^{2}} .
$$

Dividing (14') through by $\gamma_{V}$, we've, because, among other algebraic manipulations, 1 $v_{x} V / c^{2}=\frac{1}{\gamma_{V}^{2}\left(1+v_{x}^{\prime} V / c^{2}\right)}, \frac{v_{x}-V}{c^{2}}=\frac{v_{x}^{\prime} / c^{2}}{\gamma_{V}^{2}\left(1+v_{x}^{\prime} V / c^{2}\right)}$,

$$
\frac{1}{\gamma_{V}^{4}\left(1+v_{x}^{\prime} V / c^{2}\right)^{3}}\left(L_{1}^{\prime} v^{\prime}{ }_{1}\right)=0
$$

or just, $L_{1}^{\prime} v^{\prime}{ }_{1}=0$, establishing the covariance of the $m=n=1$ component of (14). Some tedious calculations show that all components are covariant but we do not pause to do that here. (It saves some effort if one realizes that the only independent calculations that need be made, besides for the $(1,1)$ component are for the $(2,2),(1,2)$ and $(2,3)$ components: $(3,3)$ is the same as $(2,2)$ and $(1,3)$ is the same as $(1,2)$.)

We next turn to the solution of (14), concentrating at first on the 1-D solution, by which we mean one dimension of space $(x)$ plus time $(t)$.

\section{The 1-D Solution; Hyperbolic Motion and Bell's Rocket Paradox.}

If all motion is confined along the $x$-axis, then, where $v:=v_{x}$, our condition of rigidity becomes,

$$
\frac{\partial v}{\partial x}+\frac{v}{c^{2}} \frac{\partial v}{\partial t}=0
$$

This is a single quasilinear partial differential equation in a single dependent variable and is solved by finding the first integrals of the ordinary differential equations,

$$
\frac{d x}{1}=\frac{d t}{v / c^{2}}=\frac{d v}{0}
$$

The first integrals are, $v=$ const., and, $\tau:=t-v x / c^{2}=$ const.. Therefore, according to the standard theory, the general solution of $\left(14^{\prime}\right)$ is, $F(v, \tau)=0$, where $F$ is an arbitrary function. Usually we can safely write,

$$
v=v(\tau)
$$

Note that this is an implicit algebraic relation due to the appearance of $v$ on the right hand side of the equation (in $\tau$ ). 
A simple instance of this leads to hyperbolic motion, studied by Misner, Thorne and Wheeler in reference [3]. Assume,

$$
v=a \tau
$$

where $a$ is a constant. Then,

$$
v=a(t-v x) / c^{2}
$$

or,

$$
v=\frac{a t}{1+a x / c^{2}}
$$

The trajectories, $x=x(t)$ are found by integrating,

$$
\frac{d x}{d t}=\frac{a t}{1+a x / c^{2}},
$$

or, $\left(1+a x / c^{2}\right) d x=a t d t$, which gives the equation of a hyperbola, explaining the name of the motion,

$$
\left(1+a x / c^{2}\right)^{2}-\left(1+a x^{\prime} / c^{2}\right)^{2}=\frac{a^{2} t^{2}}{c^{2}}
$$

where $x^{\prime}$ is the value that $x$ takes when $t=0$, when, according to (15), $v=0$ also. Thus $x^{\prime}$ is the Cartesian coordinate in the accelerated frame, $\Upsilon$, as explained in $\S 1$.

In the limit, $c \rightarrow \infty$, (15) is just $v=a t$, and (16) is, $x=x^{\prime}+\frac{1}{2} a t^{2}$, which is just classical constant acceleration.

Misner, Thorne and Wheeler [3] write (16) as,

$$
\left(\frac{a x}{c^{2}}\right)^{2}-\left(\frac{a x^{\prime}}{c^{2}}\right)^{2}=\frac{a^{2} t^{2}}{c^{2}}
$$

which is just a shift of the origin of $x$. Also, they write, $v=\frac{c^{2} t}{x}$, in place of (15), which looks funny and obscures the fact that we are just dealing with old fashion constant acceleration.

Returning to (15) and (16) as we've written them, notice that in regions where $x<$ $x_{C}(t):=c t-c^{2} / a$ that $v>c$.

Clearly, this is relativistically impossible and simply means that rigid motion cannot extend into this region and plastic flow must set in for points to the left of $x_{C}(t)$. This boundary starts out at $t=0$ at $x_{C}=-c^{2} / a$, far to the left of the origin, and thunders along to the right at speed $c$. It is a shock wave, being a wave of discontinuity in the type of motion on either 
side of the boundary (rigid vs. plastic). The trajectory $x=x_{C}(t)$ is the asymptote of the hyperbola, (16), and the velocity of the shock wave is $v=\frac{a t}{1+a x_{C}(t) / c^{2}}=c$. The asymptote $i$ s the shock front.

We conjecture that in any rigid accelerated frame regions of forced plastic flow, caused by reaching a limit where $v=c$. We will find later that a rotating frame is given by $v=\omega r$, where $\omega$ is a constant; in this case, $v=c$ when $r=c / \omega$ and this must be a shock front beyond which there is plastic flow.

Thus, a Ferris Wheel rotating with earth's $\omega_{E}$ could not be extended beyond about the radius of Neptune's orbit. The stars seem to rotate with angular velocity $-\omega_{E}$ in the night sky not because they are attached to some giant Ferris Wheel, but because it is the earth which is rotating beneath them. The stars are at rest in a reference frame which is approximately rigid, and earth is rotating with respect to this approximately inertial reference frame. Relativity acknowledges that there is something "absolute" about acceleration and rotation in that accelerated or rotated frames are not equivalent to inertial frames of reference. The presence or absence of gravity physically distinguishes accelerating frames from inertial ones per the principle of equivalence, which was Einstein's insight that allowed him to give a physical distinction between accelerated and inertial frames, as opposed to the merely geometrical distinction of Newton. (Note that the frame of reference of the stars possesses gravity and is not strictly inertial, but this gravity is not strong enough to make the space significantly nonEuclidean, so we may treat it as approximately inertial in the sense of special relativity; meanwhile the space of a rotating reference frame becomes highly non-Euclidean when $v=$ $\omega r$ reaches an appreciable fraction of $c$.)

Note that the frame of reference of the earth becomes plastic well before approaching the orbit of Neptune: while the lower regions of the earth's atmosphere rotate with the earth, the upper reaches merge continuously with the local solar wind.

In the case of the Schwarzchild solution [9] in general relativity, which is the basis of black hole theory, a region where escape velocity - the velocity of free fall from rest at infinityexceeds $c$ is inside the "Schwarzchild radius", $R_{0}:=2 G M / c^{2}$, where $G$ is the constant of Universal Gravitation and $M$ is the mass of the black hole. The Schwarzchild surface is then a shock front and not a "singularity" in space-time. The coordinate system, $(r, \theta, \varphi, t)$, of the standard form of the Schwarzchild solution describes a rigid frame, $\Upsilon$. (We know it is rigid, 
because according to a formula in ref [10], $g_{m n}=-\mathfrak{g}_{m n}+\frac{\mathfrak{g}_{m 4} \mathfrak{g}_{n 4}}{c^{2}}=-\mathfrak{g}_{m n}$ and Schwarzchild's solution is static.)

Now, $-\mathfrak{g}_{11}=\frac{1}{1-\frac{R_{0}}{r}}$, and, $\mathfrak{g}_{44}=c^{2}\left(1-\frac{R_{0}}{r}\right)$, reverse sign inside the Schwarzchild surface (for $\left.r<R_{0}\right)$. This indicates that $\Upsilon$ is not physically accessible inside that region, but that $\Upsilon$ is moving with speed $>c$ with respect to frames accessible in that region, which are the inertial frames in free fall. The inertial frames inside $R_{0}$ are receding from those on the outside by speeds in excess of $c$ and cannot be accessed by frames at rest with respect to the outside inertial frames in the inside region. Relativity postulates that matter cannot exceed speed $c$ with respect to the local $\Sigma$, but that does not mean that different parts of $\Sigma$ cannot separate from each other at speeds $>c$, and this is what is happening in the vicinity of the Schwarzchild shock front.

In special relativity, however, it is postulated that $\Sigma$ is globally rigid and Euclidean with no parts separating from others at any speed, much less one $>c$. Therefore a region in which a rigid $\Upsilon$ is inaccessible is simply a region into which rigid motion cannot be continued in the first place.

Note that in the frame, $\Upsilon$, of hyperbolic motion, we have (ref [3]), $d \sigma^{2}=-d x^{\prime 2}+c^{2}(1+$ $\left.\frac{a x^{\prime}}{c^{2}}\right)^{2} d t^{\prime 2}$, and $\left(1+\frac{a x^{\prime}}{c^{2}}\right)^{2}=\left(1+\frac{a x}{c^{2}}\right)^{2}-\frac{a^{2} t^{2}}{c^{2}}<0$ when $x<c t-c^{2} / a$, so $g_{44}<0$ in this region behind the shock front, and because $1+\frac{a x^{\prime}}{c^{2}}$ is imaginary, $d x^{\prime 2}<0$ and $g_{11}<0$ in this region. It is altogether analogous to the situation with the Schwarzchild solution inside $R_{0}$ : both $g_{11}=\mathfrak{g}_{11}$ and $\mathfrak{g}_{44}$ reverse signs behind the shock front.

A less competent commentator might conclude that space and time had "reversed polarity" inside these regions, but it really just indicates that reference frames accessible in these regions cannot be rigid continuations of the $\Upsilon$ which exists in the subluminal regions, that the rigid continuations are not physically accessible.

A similar circumstance occurs for regions of rotating frames for which $r>c / \omega$.

I am willing to conjecture that all solutions to relativistic gravitation are, generally speaking, isomorphic with the hyperbolic motion solution of special relativity.

After soaring to such astral heights discussing black holes and general relativity, let us return to the terra firma of the special theory. 
We conclude this section by discussing the notorious Bell rocket paradox.

Bell [11] considered the problem of two rockets accelerating along the $x$-axis in such a way that a string connecting them would not break or go slack. He concluded that the rockets would have to get closer over time as judged by an observer in $\Sigma$ with respect to whom they were accelerating; that is, the two rockets do not move with the same velocity at the same time: the rearward one moves faster and gets closer to the forward one over time, even though the string does not go slack, which may seem paradoxical. But there is no paradox.

What Bell is describing is a system which is rigid as judged by itself (the string does not break or go slack) - i.e. an accelerating rigid reference frame. That is, the distance, $d s^{\prime}$, between the forward and rearward rockets is constant in their reference frame, $\Upsilon$. Thus, by way of illustration, if we assume the rockets are undergoing constant acceleration, $a>0$, and are moving along the $x>0$ axis as judged by the observing $\Sigma$ with velocities, $v(x)>0$, then $v(x)$, the velocity of the rocket at position $x$ in $\Sigma$ must be given by (15). Then if, $\left(x_{R}, v_{R}\right)$, describe the rearward rocket and, $\left(x_{F}, v_{F}\right)$, describe the forward rocket, then by (15) clearly, $v_{F}<v_{R}$ if $x_{F}>x_{R}$, and so the rearward rocket will seem to catch up to the forward rocket in $\Sigma$ and the string will seem to shorten. In fact this happens for any accelerating system, not just

one with a constant $a$, because, from (14"), $\frac{\partial v}{\partial x}=-\frac{v}{c^{2}} \frac{\partial v}{\partial t}<0$ if $\frac{\partial v}{\partial t}>0$ and $v>0$. And this is not paradoxical at all because the Lorentz contraction of the string depends on its velocity with respect to $\Sigma$ and shortens as the velocity accelerates: the rearward rocket must move at a higher speed than the forward rocket to catch up with it and shorten the string.

We have thus learned the general lesson that a frame which judges itself to be rigid may appear not to be rigid as judged by another rigid frame. This is paradoxical only in the sense that classical theory does not set us up for such an expectation. But, as we've just seen, it is not at all paradoxical.

Let us move on to consideration of the general 3-D case where, inter alia, rotation may occur.

\section{The 3-D Solution.}

Recall that in the classical case, rigid motion is governed by (8); in this case, define a rotation tensor,

$$
\omega_{m n}:=\frac{1}{2}\left(\frac{\partial v_{m}}{\partial x_{n}}-\frac{\partial v_{n}}{\partial x_{m}}\right)
$$


which, together with (8), yields,

$$
\frac{\partial v_{m}}{\partial x_{n}}=\omega_{m n}
$$

Note, from (17), the rotation matrix is antisymmetric and its diagonal elements are zero.

Consider, $\frac{\partial \omega_{12}}{\partial x_{1}}=\frac{\partial^{2} v_{1}}{\partial x_{2} \partial x_{1}}=0$, because the $(1,1)$ component of $(18)$ is zero; likewise $\frac{\partial \omega_{12}}{\partial x_{2}}=-$ $\frac{\partial \omega_{21}}{\partial x_{2}}=-\frac{\partial^{2} v_{2}}{\partial x_{1} \partial x_{2}}=0$, and

$$
\frac{\partial \omega_{12}}{\partial x_{3}}=\frac{\partial \omega_{13}}{\partial x_{2}}=-\frac{\partial \omega_{31}}{\partial x_{2}}=-\frac{\partial \omega_{32}}{\partial x_{1}}=\frac{\partial \omega_{23}}{\partial x_{1}},
$$

while, $-\frac{\partial \omega_{21}}{\partial x_{3}}=-\frac{\partial \omega_{23}}{\partial x_{2}}$; therefore, because, $\frac{\partial \omega_{12}}{\partial x_{3}}=-\frac{\partial \omega_{21}}{\partial \omega_{3}}$, we need, $\frac{\partial \omega_{23}}{\partial x_{1}}=-\frac{\partial \omega_{23}}{\partial x_{1}}=0$. Therefore, wrapping up, we've, $\frac{\partial \omega_{m n}}{\partial x_{r}}=0$, for any $(m, n, r)$, and $\omega_{m n}=\omega_{m n}(t)$ is a function of $t$ alone.

Therefore, integrating (18) yields, where $v_{0 m}(t)$ is a "constant" of integration,

$$
v_{m}(\boldsymbol{x}, t)=v_{0 m}(t)+\omega_{m n} x_{n}
$$

or, defining a rotation vector, $\omega_{m}=\omega_{m^{\prime} m^{\prime \prime}}$, with $\left(m, m^{\prime}, m^{\prime \prime}\right)$ cyclic in $(1,2,3)$, we've,

$$
\boldsymbol{v}(\boldsymbol{x}, t)=\boldsymbol{v}_{0}(t)+\boldsymbol{\omega} \times \boldsymbol{x}
$$

This is the most general classical rigid motion: translation $\left(\boldsymbol{v}_{0}\right)$ plus rotation $(\boldsymbol{\omega} \times \boldsymbol{x})$.

Now we pose the classical "trajectory problem", or " $C_{0}$ problem": suppose that there is a point, $\boldsymbol{x}_{0}$, of the rigid body which moves along a trajectory, $C_{0}$, parameterized as, $\boldsymbol{x}_{0}(t)$, and suppose that as the body moves along this trajectory, the body rotates around that point with angular velocity, $\boldsymbol{\omega}(t)$. We claim that the specification of this initial condition or boundary condition completely determines the rigid motion of the entire body and it should be intuitively clear that this is so; however, we now pause to state the simple proof.

Rewrite (19) as,

$$
\boldsymbol{v}(\boldsymbol{x}, t)=\boldsymbol{v}_{0}(t)+\boldsymbol{\omega}(t) \times \boldsymbol{x}_{0}(t)+\boldsymbol{\omega}(t) \times\left(\boldsymbol{x}-\boldsymbol{x}_{0}(t)\right) .
$$

In this solution, the translational velocity of $\boldsymbol{x}_{0}$ is $\boldsymbol{v}_{0}(t)+\omega(t) \times \boldsymbol{x}_{0}(t)$ and the rotational velocity about that point (zero at $\boldsymbol{x}_{0}$ by definition) is the remaining term in (20).

Let us now turn to the relativistic case. Define the relativistic rotation matrix as, 


$$
\omega_{m n}:=\frac{1}{2}\left(L_{n} v_{m}-L_{m} v_{n}\right)
$$

then, together with (14), we've,

$$
L_{n} v_{m}=\omega_{m n}
$$

as our new expression of the rigidity condition.

Let us make the following transformation of variables,

$$
\begin{array}{ll}
\tau=t-v_{r} x_{r} / c^{2}, & s_{n}=x_{n}, \\
t=\tau+v_{r} s_{r} / c^{2}, & x_{n}=s_{n} .
\end{array}
$$

Then,

$$
\begin{aligned}
& \frac{\partial}{\partial x_{m}}=\frac{\partial}{\partial s_{m}}-\left(\frac{v_{m}}{c^{2}}+x_{r} \frac{\partial v_{r}}{\partial x_{m}} / c^{2}\right) \frac{\partial}{\partial \tau}, \\
& \frac{\partial}{\partial t}=\left(1-x_{r} \frac{\partial v_{r}}{\partial t} / c^{2}\right) \frac{\partial}{\partial \tau}, \\
& L_{m}=\frac{\partial}{\partial s_{m}}-\frac{s_{r} \omega_{m r}}{c^{2}} \frac{\partial}{\partial \tau},
\end{aligned}
$$

then, when $\omega_{m n}=0$, the rigidity condition, (22), becomes,

$$
\left.\frac{\partial v_{n}}{\partial s_{m}}\right|_{\tau}=0 .
$$

That is, when expressed as a function of $s_{m}$ and $\tau, v_{m}$ is a function of $\tau$ alone in the irrotational case, just as we found in the one-dimensional case.

The rotational case requires a separate treatment and we turn to that in $\S 6$; meanwhile, in the remainder of this section, we consider the trajectory $/ C_{0}$ problem.

If a single point of the rigid body is known to move irrotationally along a curve, $C_{0}$, parameterized by $t$, i.e.: $\boldsymbol{x}=\boldsymbol{x}_{0}(t), \boldsymbol{v}=\boldsymbol{v}_{\mathbf{0}}(t)=\dot{\boldsymbol{x}}_{0}(t)$, what is the full motion of the rigid body at all points in space and time of $\Sigma$ ?

One approach-let us call it the "formal approach"-is to state $v$ as a given function of $\tau$ and then $v(x, t)$ can immediately be inferred from $v_{n}(\tau)$, with $\tau=t-v_{r} x_{r} / c^{2}$, as can the trajectory of any point in the body by integrating,

$$
\frac{d \boldsymbol{x}}{d t}=\boldsymbol{v}(\tau)
$$

that is, 


$$
\frac{d x}{d \tau}=\frac{v(\tau)\left(1+\boldsymbol{v}^{\prime}(\tau) \cdot x / c^{2}\right)}{1-v^{2}(\tau) / c^{2}}
$$

because, $\frac{d}{d t}=\frac{d \tau}{d t} \frac{d}{d \tau}$, and, $\frac{d \tau}{d t}=\frac{1-v^{2}(\tau) / c^{2}}{1+v^{\prime}(\tau) \cdot x / c^{2}}$.

Note that (23) is a coupled system of linear nonhomogeneous ordinary differential equations, viz:

$$
\frac{d \boldsymbol{x}}{d \tau}-\frac{\boldsymbol{v}(\tau) / c^{2}}{1-v^{2}(\tau) / c^{2}} \boldsymbol{v}^{\prime}(\tau) \cdot \boldsymbol{x}=\frac{\boldsymbol{v}(\tau)}{1-v^{2} / c^{2}}
$$

This system of equations is analytically solvable in principle (though in practice, with the current state of mathematical technique, it may require some use of numerical methods) because the $v(\tau)$ are given functions of $\tau$. The solution involves three constants of motion (constants of integration), clearly the coordinates of a particular point of the body in the coordinate system, $\boldsymbol{u}$, of $\Upsilon$, the reference frame in which the body is at rest. There is some degree of arbitrariness in the definition of $\boldsymbol{u}$ of course, but because the motion is irrotational and $\Upsilon$ is Euclidean, we may choose Cartesian coordinates. If $\Upsilon$ is momentarily at rest with respect to $\Sigma$ at some time, $t_{0}$, we may simply choose $\boldsymbol{u}:=\boldsymbol{x}^{\prime}=\boldsymbol{x}\left(t_{0}\right)$, the Cartesian coordinate of the corresponding point in $\Sigma$ at time $t_{0}$, which is what we did in $\S 4$. The trajectory, $C_{0}$, is thus given implicitly in the formal approach.

The direct trajectory $/ C_{0}$ approach is to state $C_{0}: \boldsymbol{x}=\boldsymbol{x}_{0}(t), \boldsymbol{v}=\boldsymbol{v}_{0}(t)=\dot{\boldsymbol{x}}_{0}(t)$ explicitly and solve in the following way:-

Because, $\tau=t-\boldsymbol{v}_{0}(t) \cdot \boldsymbol{x}_{0}(t) / c^{2}$, this equation may be inverted (analytically in principle, possibly numerically in practice) to give, $t=t(\tau)$. Then,

$$
\boldsymbol{v}=\boldsymbol{v}(\tau)=\boldsymbol{v}(t(\tau))
$$

which states $v$ as a given function of $\tau$ and we may proceed from there by using the formal technique. Two simple examples will be given in $§ 7$.

Meanwhile we turn to rotation.

\section{Relativistic Rotation.}

Let us consider the commutation relations of the two most prominent differential operators in the theory, $L_{m}$ and $\frac{\partial}{\partial t}$ (where the commutator is $[A, B]=A B-B A$ ). We have at once by direct calculation:- 


$$
\begin{aligned}
& {\left[L_{m}, L_{n}\right]=\frac{2 \omega_{m n}}{c^{2}} \frac{\partial}{\partial t},} \\
& {\left[L_{m}, \frac{\partial}{\partial t}\right]=-\frac{a_{m}}{c^{2}} \frac{\partial}{\partial t},}
\end{aligned}
$$

where $a_{m}:=\frac{\partial v_{m}}{\partial t}($ not the total derivative).

Applying (25) to $v_{n}$, we obtain,

$$
L_{m} a_{n}-\frac{\partial \omega_{m n}}{\partial t}=-\frac{a_{m} a_{n}}{c^{2}}
$$

Applying (24) to $v_{n}$ (no sum on $n$ ), because $L_{n} v_{n}=0$, yields,

$$
L_{n} \omega_{n m}=-\frac{2 \omega_{n m} a_{n}}{c^{2}}
$$

Similarly, because $\omega_{n m}=-\omega_{m n}$, we've,

$$
L_{m} \omega_{n m}=-\frac{2 \omega_{n m} a_{m}}{c^{2}} .
$$

Applying (24) to $\omega_{n m}$ (no sum on $m$ or $n$ ), we obtain,

$$
L_{m} L_{n} \omega_{n m}-L_{n} L_{m} \omega_{n m}=\frac{2 \omega_{n m}}{c^{2}} \frac{\partial \omega_{n m}}{\partial t} .
$$

With use of (26), (27) and (27'), we see that (28) reduces to,

$$
-\frac{4}{c^{2}} \omega_{n m} \frac{\partial \omega_{n m}}{\partial t}=\frac{2}{c^{2}} \omega_{n m} \frac{\partial \omega_{n m}}{\partial t}
$$

or,

$$
\frac{6}{c^{2}} \omega_{n m} \frac{\partial \omega_{n m}}{\partial t}=0
$$

Note that when $c \rightarrow \infty$ this is just $0=0$ and there is no restriction, but in the relativistic case we need either $\omega_{m n}=0$ or $\frac{\partial \omega_{m n}}{\partial t}=0$, but if the former is satisfied, then the latter is automatically, so the only real restriction is,

$$
\frac{\partial \omega_{m n}}{\partial t}=0 .
$$

This is required by the argument of Ehrenfest mentioned in the Introduction.

Because of (29'), equation (26) becomes,

$$
L_{m} a_{n}=-\frac{a_{m} a_{n}}{c^{2}} .
$$

Applying $L_{r}$ to (26'), we have, 


$$
L_{r} L_{m} a_{n}=\frac{2 a_{m} a_{n} a_{r}}{c^{4}}
$$

similarly, $L_{m} L_{r} v_{n}=\frac{2 a_{m} a_{n} a_{r}}{c^{4}}$, or, $\left[L_{m}, L_{r}\right] a_{n}=0$, or, from (24),

$$
\frac{2 \omega_{r m}}{c^{2}} \frac{\partial a_{n}}{\partial t}=0
$$

If $\omega_{r m}=0$, there is no restriction and we have the theory of $\S 5$, but if there is rotation, we have,

$$
\frac{\partial a_{n}}{\partial t}=0
$$

Next, we turn to the general solution of our rigidity condition, (22), under the restrictions, $\left(29^{\prime}\right)$ and $\left(30^{\prime}\right)$ for nonzero rotation.

Since $\boldsymbol{a}=\frac{\partial v}{\partial t}$ must be independent of $t$, we may write,

$$
v_{n}=a_{n}(\boldsymbol{x}) t+b_{n}(\boldsymbol{x})
$$

Thus,

$$
\omega_{r n}=L_{n} v_{r}=\frac{\partial a_{r}}{\partial x_{n}} t+\frac{\partial b_{r}}{\partial x_{n}}+\frac{a_{n} t+b_{n}}{c^{2}} a_{r}
$$

which must be independent of $t$, therefore we have,

$$
\begin{aligned}
& \frac{\partial a_{r}}{\partial x_{n}}+\frac{a_{r} a_{n}}{c^{2}}=0, \\
& \frac{\partial b_{r}}{\partial x_{n}}+\frac{b_{n} a_{r}}{c^{2}}=\omega_{r n} .
\end{aligned}
$$

Note that (31) is equivalent to $\left(26^{\prime}\right)$ because of $\left(30^{\prime}\right)$.

Consider the $n=r$ components of these two equations (no summing); since $\omega_{r r}=0$, we have,

$$
\begin{aligned}
& \frac{\partial a_{r}}{\partial x_{r}}+\frac{a_{r} a_{r}}{c^{2}}=0 \\
& \frac{\partial b_{r}}{\partial x_{r}}+\frac{b_{r} a_{r}}{c^{2}}=0 .
\end{aligned}
$$

Write (31') as,

$$
\frac{d a_{r}}{a_{r}^{2}}=-\frac{d x_{r}}{c^{2}},
$$

where $x_{r^{\prime}}$ and $x_{r^{\prime \prime}}$ can be regarded as constants of integration $\left(\left(r, r^{\prime}, r^{\prime \prime}\right)\right.$ cyclic in $\left.(1,2,3)\right)$, thus, 


$$
a_{r}=\frac{1}{\frac{x_{r}}{c^{2}}+f_{r}\left(x_{r^{\prime}}, x_{r^{\prime \prime}}\right)}
$$

where $f_{r}$ is the "constant" of integration. This formula is the same with $r, r$ ' and $r$ " replacing each other cyclically, so with some algebraic manipulation, we arrive at,

$$
a_{r}=\frac{k_{r}}{\mu}
$$

where $k_{r}$ are constants and,

$$
\mu:=1+\left(k_{1} x_{1}+k_{2} x_{2}+k_{3} x_{3}\right) / c^{2}
$$

Similar manipulations of (32) and (32') yield,

$$
b_{r}=\frac{g_{r}\left(x_{\left.r^{\prime}, x_{r}^{\prime \prime}\right)}\right)}{\mu}
$$

where,

$$
g_{r}=\Omega_{r r^{\prime}} x_{r^{\prime}}+\Omega_{r r^{\prime \prime}} x_{r^{\prime \prime}}+c_{r}
$$

where $\Omega_{m n}$ is an antisymmetric matrix of constants (diagonal elements of course zero) and $c_{r}$ are constants.

Thus, in sum, we have,

$$
\omega_{m n}=\frac{\Omega_{m n}}{\mu}+\frac{k_{m} g_{n}-k_{n} g_{m}}{\mu^{2} c^{2}}
$$

Usually one introduces a rotation vector, $\omega_{r}$, defined, in our notation as $-\omega_{r^{\prime} r^{\prime \prime}}$.

Note that, although $\boldsymbol{\omega}$ is independent of $t$, it is not independent of position, $\boldsymbol{x}$. For instance, if at the origin, $\boldsymbol{\omega}$ is pointed in the $z$-direction $\left(\omega_{1}=\omega_{2}=0\right)$ and there is a nonzero acceleration, $k_{3}$, along the $z$-axis at the origin, then $\omega_{1}$ and $\omega_{2}$ will take nonzero values away from the origin and the axis of rotation will deviate from the $z$-axis.

The equations, (33)-(36), together with,

$$
\frac{d x_{r}}{d t}=a_{r} t+b_{r}
$$

give a system of coupled linear nonhomogeneous ordinary differential equations which can be solved analytically in principle, though in practice numerical methods may be required. There are three constants of motion/integration, which are related to the coordinates, $\boldsymbol{u}$, of a point fixed in $\Upsilon$; this time, however, owing to the fact that $\Upsilon$ must be non-Euclidean due to 
the fact that $\Upsilon$ is rotating, we must be content with curvilinear $\boldsymbol{u}$ in the definition of which there is a wide degree of arbitrariness. This makes sense, because we are free to use any coordinate system we want to in order to label the points of a space. Choosing, for instance, $\boldsymbol{u}=(0,0,0)$, gives the initial trajectory in an implicit way in a manner similar to the "formal approach" to the irrotational case.

The approach just considered — stating the constants, $\boldsymbol{k}, \boldsymbol{c}$ and $\Omega$, and deriving $C_{0}$ - could be called the formal approach for the rotational case. The inverse problem-stating $C_{0}$ and a rotation about the point moving along it and deriving the constants-would be complicated by the fact that not just any $\boldsymbol{\omega}$ would be allowed, but only one leading to an acceptable solution by the formal approach. It would not be as simple as stating that $\boldsymbol{\omega}$ be constant along $C_{0}$ because it may have a nonzero $\frac{d \omega}{d t}$ even though $\frac{\partial \omega}{\partial t}$ is zero. This final little technicality I leave as an open problem.

\section{Hyperbolic Motion and Revolution Without Rotation.}

Finally, let us conclude this article by considering two simple examples of irrotational rigid motion.

First, suppose we are given as $C_{0}$ in one dimension,

$$
x_{0}+\frac{1}{2} \frac{a x_{0}^{2}}{c^{2}}=\frac{1}{2} a t^{2} .
$$

This is obviously contrived, but we wish to illustrate technique. This gives,

$$
x_{0}=\frac{-1+\sqrt{1+\frac{a^{2} t^{2}}{c^{2}}}}{a / c^{2}}
$$

which is approximately $x_{0}=\frac{1}{2} a t^{2}$, or our familiar problem of constant acceleration (hyperbolic motion).

Differentiating,

$$
v_{0}=\dot{x}_{0}=\frac{a t}{\sqrt{1+a^{2} t^{2} / c^{2}}}
$$

Thus, 


$$
\tau=t-v_{0} x_{0} / c^{2}=\frac{t}{\sqrt{1+a^{2} t^{2} / c^{2}}}
$$

which, upon inverting, gives,

$$
t=\frac{\tau}{\sqrt{1-a^{2} \tau^{2} / c^{2}}} .
$$

Therefore, the complete solution is,

$$
v=v_{0}(t(\tau))=\frac{a \tau / \sqrt{1-a^{2} \tau^{2} / c^{2}}}{\sqrt{1+\frac{a^{2} \tau^{2} / c^{2}}{1-a^{2} \tau^{2} / c^{2}}}}=a \tau,
$$

which we recognize as hyperbolic motion, for which we can solve for the trajectories as in $\S 4$ (see (15), (16)), or as follows:-

Recall (23), which here becomes,

$$
\frac{d x}{d \tau}=\frac{a \tau\left(1+a x / c^{2}\right)}{1-a^{2} \tau^{2} / c^{2}}
$$

or,

$$
\frac{d x}{1+a x / c^{2}}=\frac{\frac{1}{2} a d\left(\tau^{2}\right)}{1-a^{2} \tau^{2} / c^{2}}
$$

or,

$$
\left(1+\frac{a^{2} x^{2}}{c^{2}}\right)^{2}\left(1-\frac{a^{2} \tau^{2}}{c^{2}}\right)=\text { const }
$$

If $x=x^{\prime}$ when $\tau=0$, then const. $=\left(1+\frac{a^{2} x^{\prime 2}}{c^{2}}\right)^{2}$. Now, $v=a \tau=a\left(1-v x / c^{2}\right)$, or $v=$ $\frac{a t}{1+a x / c^{2}}$, and $\tau=\frac{v}{a}=\frac{t}{1+a x / c^{2}}$, therefore, $1-\frac{a^{2} \tau^{2}}{c^{2}}=1-\frac{a^{2} t^{2} / c^{2}}{\left(1+a^{2} x^{2} / c^{2}\right)^{2}}$, and we once again arrive at (16).

Lastly consider the case of revolution at angular rate $\Omega$ without rotation, which we will solve using (23).

The trajectory $C_{0}$ is given by,

$$
x_{0}=R \cos \Omega t
$$




$$
y_{0}=R \sin \Omega t
$$

where $R$ and $\Omega$ are constants. Then,

$$
\begin{aligned}
& v_{0 x}=-\Omega R \sin \Omega t, \\
& v_{0 y}=\Omega R \cos \Omega t .
\end{aligned}
$$

We illustrate first the classical case; $\boldsymbol{v}$ is a function of $t$ alone, therefore, $v_{x}=v_{0 x}$, or,

$$
\frac{d x}{d t}=-\Omega R \sin \Omega t
$$

similarly,

$$
\frac{d y}{d t}=\Omega R \cos \Omega t
$$

and thus,

$$
\begin{gathered}
x=x^{\prime}-R+R \cos \Omega t, \\
y=y^{\prime}+R \sin \Omega t
\end{gathered}
$$

where $(x, y)=\left(x^{\prime}, y^{\prime}\right)$ when $t=0$.

Returning to the relativistic case, on $C_{0}$,

$$
\tau=t-\boldsymbol{v}_{0} \cdot \boldsymbol{x}_{0} / c^{2}=t
$$

therefore

$$
\boldsymbol{v}=\boldsymbol{v}(\tau)=\boldsymbol{v}_{0}(t(\tau))=-\Omega R \sin \Omega \tau \boldsymbol{i}+\Omega R \cos \Omega \tau \boldsymbol{j}
$$

Thus, away from $C_{0}$,

$$
\tau=t-\boldsymbol{v} \cdot x / c^{2}=t+\frac{\Omega R x}{c^{2}} \sin \Omega \tau-\frac{\Omega R y}{c^{2}} \cos \Omega \tau
$$

If we could analytically solve this implicit equation for $\tau=\tau(t, x, y)$ we could substitute it in the expression just given for $\boldsymbol{v}(\tau)$ and integrate the system $\frac{d \boldsymbol{x}}{d t}=\boldsymbol{v}(t, \boldsymbol{x})$, but the prospect would be daunting because this system is not necessarily linear; therefore it is desirable to use the method based on (23) which, though difficult, is linear.

By way of comparison, in the case of hyperbolic motion, we had $v=a \tau$ and $\tau=t-a \tau x / c^{2}$, so $\tau=\frac{t}{1+a x / c^{2}}$ and $\frac{d x}{d t}=v(\tau)=a \tau=\frac{a t}{1+a x / c^{2}}$, which proved to be readily integrable; so in the 
hyperbolic case both methods were equally simple. However, in the case of revolution we are not so lucky and only one of the methods is relatively feasible.

\section{The Meaning of the Variable $\tau$.}

In the irrotational case, we find that

$$
\boldsymbol{v}=\boldsymbol{v}(\tau)
$$

where,

$$
\tau=t-v \cdot x / c^{2}
$$

Since $\boldsymbol{v}=$ const. when $\tau=$ const., this can be written,

$$
\boldsymbol{v}=\boldsymbol{v}\left(\tau^{\prime}\right)
$$

with,

$$
\tau^{\prime}=\frac{t-v \cdot x / c^{2}}{\sqrt{1-v^{2} / c^{2}}}
$$

Now, (39) is reminiscent of the standard form of the Lorentz transformation when $\boldsymbol{v}$ is constant, while (38) is reminiscent of the classical $\boldsymbol{v}=\boldsymbol{v}(t)$ in the irrotational case, so we might be tempted to regard $\tau^{\prime}$ as the time in an accelerated non-rotating frame, $\Upsilon$. However, this does not coincide with the proper time or the reading on a clock moving along a trajectory which is a point at rest in $\Upsilon$. According to the usual formula of relativity, this would be given by,

$$
\left.\frac{d t^{\prime}}{d t}\right|_{x^{\prime}}=\sqrt{1-v^{2} / c^{2}}
$$

where $x^{\prime}$ is the spatial coordinate at rest in $\Upsilon$; to hold $x^{\prime}$ constant is to follow the trajectory of a point fixed in $\Upsilon$.

Let us illustrate by considering the 1-D case of hyperbolic motion. Recall (15) and (16). Performing the integration,

$$
t^{\prime}=\int \sqrt{1-v^{2} / c^{2}} d t
$$

holding $x^{\prime}$ constant in (16), we obtain,

$$
t^{\prime}=\frac{1+a x^{\prime} / c^{2}}{a / c} \sinh ^{-1} \frac{a t / c}{1+a x^{\prime} / c^{2}}
$$


This is not the $\tau^{\prime}$ of (39), nor is it $\tau$ which $=v / a$, and on using (15) and (16), we've,

$$
\tau=\frac{t}{1+a x / c^{2}}=\frac{c}{a} \tanh \frac{a t^{\prime} / c}{1+a x^{\prime} / c^{2}}
$$

which is only approximately equal to $t^{\prime}$ for low values of $t^{\prime}, a$ and $x^{\prime}$.

Now,

$$
\sqrt{1-v^{2} / c^{2}}=\sqrt{1-a^{2} \tau^{2} / c^{2}}=\operatorname{sech} \frac{a t^{\prime} / c}{1+a x^{\prime} / c^{2}},
$$

so,

$$
\tau^{\prime}=\frac{\tau}{\sqrt{1-v^{2} / c^{2}}}=\frac{c}{a} \sinh \frac{a t^{\prime} / c}{1+a x^{\prime} / c^{2}} .
$$

In fact, we see from this and (42) that,

$$
\tau^{\prime}=\frac{t}{1+a x^{\prime} / c^{2}}=\frac{t}{\sqrt{\left(1+a x / c^{2}\right)^{2}-a^{2} t^{2} / c^{2}}} .
$$

So, matters stand as follows:-

(1) Expressed as a function of $\tau$ or $\tau^{\prime}$ and $x, v$ is a function of $\tau$ (or $\tau^{\prime}$ ) alone.

(2) The reading on a moving clock is neither $\tau$ nor $\tau^{\prime}$ but $t^{\prime}$.

(3) Expressed as a function of $t^{\prime}$ and $x^{\prime}, v$ is not a function of $t^{\prime}$ alone.

(4) Expressed as a function of $t$ and $x, v$ is not a function of $t$ alone.

Thus, the analogy originally anticipated with the classical irrotational case is thoroughly dispelled.

Note, too, that $\tau$ has no particular physical meaning, but is simply the result of a convenient mathematical transformation; only $t$ and $t^{\prime}$ can be directly associated with the readings on clocks (one in $\Sigma$, the other in $\Upsilon$ ) and deserve to be called "time".

Note, lastly, the similarity between (42) and a formula in ref [3], which can here be translated as,

$$
t_{M T W}^{\prime}=\frac{c}{a} \sinh ^{-1} \frac{a t / c}{1+a x^{\prime} / c^{2}}
$$

This does not correspond to (42) because $t_{M T W}^{\prime}$ is not the proper time or the reading on a clock except along the trajectory corresponding to $x^{\prime}=0$, i.e. along $C_{0}$, which is all that Misner, Thorne and Wheeler claim it to be if one follows their derivation carefully. 
The time coordinate, $t^{\prime}{ }_{M T W}$, used by Misner, Thorne and Wheeler is chosen to make the metric, $\mathfrak{g}_{\mu \nu}$, diagonal - the usual way of doing business in the standard literature. Thus one obtains,

$$
d \sigma^{2}=-d x^{\prime 2}-d y^{\prime 2}-d z^{\prime 2}+c^{2}\left(1+\frac{a x^{\prime}}{c^{2}}\right)^{2} d t_{M T W^{\prime}}
$$

Here,

$$
\mathfrak{g}_{44}=c^{2}\left(1+\frac{a x^{\prime}}{c^{2}}\right)^{2}
$$

rather than simply $c^{2}$, which indicates that $t^{\prime}{ }_{M T W}$ is not the reading on a clock (unless $x^{\prime}=0$ ) and does not deserve to be called "the time in $\Upsilon$ ". Though in later parts of ref [3], great lengths are gone to in order to interpret the "physical meaning" of $t^{\prime}{ }_{M T W}$, the argument is awkward and feels funny. In fact it has no physical meaning as such, but, like $\tau$ or $\tau^{\prime}$, is merely a conveniently defined mathematical variable that is useful for some purposes.

\section{Conclusion.}

In this paper, by forgoing the powerful 4-D mathematics usually associated with relativity, we have attempted to illustrate the continuity between classical and relativistic concepts and methods. It is our stubborn belief that there is such continuity and the relation between space and time in relativity is simply more physically interactive, dynamic and "fluid" than the classical, "absolute" conceptions of Newton, which were laid out in the first place only as an expedient.

In our approach, $u_{4}=t$ is always the reading on a clock, while the (generally curvilinear) coordinates, $\boldsymbol{u}$, serve to distinguish different points of space, i.e., different trajectories or observers. The variable $t$ is the reading on a clock, the variables $u_{m}$ are labels that serve to distinguish an individual clock and may be thought of as some identifying mark such as a serial number.

We try, therefore, to give space and time coordinates direct physical meaning, per the original intention (followed by every theory of physics except, for some obscure reason, general relativity), rather than hyperopically treating time and space as an inseparable whole in which coordinates are merely "convenient overlays on the physical problem at hand". This 
does not mean we must forswear the use of convenient mathematical transformations of the physically meaningful variables - but that we should regard such transformed quantities as mere gimmicks rather than bestowing them with some occult physical significance, such as Misner, Thorne and Wheeler attempt to give the $t_{M T W}^{\prime}$ discussed at the end of $\S 8$.

Let us reiterate: the symbol $t$ or $u_{4}$ should be used exclusively to describe the point that a specific observer has reached in his journey (along a trajectory), i.e., the reading on his clock, or his "time", while the coordinates $u_{m}$ serve as a label to distinguish an individual observer among many in a given reference frame. A particle flying through this reference frame, thereby visiting different observers, $\boldsymbol{u}$, is an observer in its own reference frame, with its own clock reading, $t^{\prime}$, and its own clock label or serial number, $\boldsymbol{u}^{\prime}$.

In this paper we have attempted to illustrate this approach to the problem of rigid motion, i.e., to the proper handling of spatial distance relations in a general (possibly non-rigid) moving frame of reference, $\Upsilon$. The complementary problem of the proper treatment of time- hinted at in the discussion in $\S 8$ - and the arbitrariness of synchrony definitions using these techniques belongs, properly, to the topic of another paper.

\section{REFERENCES}

[1] Born, M., Ann. Phys., 30, 1, 1909; Phys. Z., 11, 233, 1910

[2] Ehrenfest, P., Phys. Z., 10, 918, 1909

[3] Misner, C. W., Thorne, K. S., Wheeler, J. A., Gravitation, Freeman, New York, 1973

[4] Bona, C., Phys. Rev. D, 27, 6, 1243, 1983

[5] Rizzi, G., Ruggiero, M. L., Eds., Relativity in Rotating Frames, Springer, Berlin, 2004

[6] Synge, J. L., Relativity: The Special Theory, $3^{\text {rd }}$ Ed., North Holland, Amsterdam, 1972

[7] Einstein, A. E., The Meaning of Relativity, Princeton University, Princeton, 1956

[8] Tsamparlis, M., Special Relativity, Springer, Heidelberg, 2010

[9] Bergmann, P. G., Introduction to the Theory of Relativity, Prentice Hall, New York, 1946

[10] Tolman, R. C., Relativity, Thermodynamics and Cosmology, Clarendon Press, Oxford, 1934

[11] Bell, J. S., Speakable and Unspeakable in Quantum Mechanics, $2^{\text {nd }}$ Ed., Cambridge University Press, Cambridge, 1987 